\title{
Localization Integrity for Intelligent Vehicles through Fault Detection and Position Error Characterization
}

\author{
Joelle Al Hage ${ }^{1}$, Philippe $\mathrm{Xu}^{1}$, Philippe Bonnifait ${ }^{1}$ and Javier Ibanez-Guzman ${ }^{2}$
}

\begin{abstract}
Localization integrity consists in providing a realtime measure of the level of trust to be placed in the localization estimates as vehicles operate. It provides a means of knowing whether position estimates are usable for navigation purposes. This paper formalizes the integrity concept and its underlying principles. Vehicles operate in different navigation environments, and so multiple sensors are used to ensure the required performance. Different sources of error exist. They must be bounded according to the acceptable level of risk for the application.

This paper presents a generic approach for addressing integrity. It combines measurement rejection (for measurements considered to be faults) and position error characterization. For this purpose, a multi-sensor data fusion with a Fault Detection and Exclusion algorithm is constituted using a bank of information filters. These filters allow detected faults to be isolated without any prior assumption regarding the number of simultaneous errors. In addition, external integrity is expressed as a Protection Level of the localization solution. It uses a Student's $t$-distribution in order to bound the distribution of the position error applicable to small integrity risks after a learning step. The approach is tested on data acquired on public roads using an experimental vehicle equipped with off-the-shelf proprioceptive and exteroceptive sensors together with an HD map. The results obtained validate the proposed approach.
\end{abstract}

Index Terms-fault detection and exclusion, error characterization, protection level computation, map aided localization.

\section{INTRODUCTION}

For mobile platforms, knowing their location is essential for navigation purposes. To increase the accuracy of localization solutions, multi-sensor data fusion methods provide good results. Higher levels of vehicle autonomy not only need higher accuracy, but also an ability to estimate error bounds in order to address uncertainty in the localization estimates in real time. The integrity concept includes this second feature. It is defined as the measure of trust on the correctness of the location estimates applied to navigation systems [1]-[3].

The accuracy of GNSS-based absolute positioning systems is improved through the fusion with multiple sources of information. For road vehicles, their location is established with respect to a map that incorporates a stored geometric description of the roads [4]. The estimated positions are associated with road maps via the map matching process [5], [6]. Information from exteroceptive sensors (e.g., cameras) is used to detect navigation feature landmarks. These are georeferenced and stored in the map to enhance the positioning output [7].

\footnotetext{
${ }^{1}$ Heudiasyc, UMR 7253, Université de Technologie de Compiègne, France. ${ }^{2}$ Renault S.A. 1 Av. Du Golf, 78064 Guyancourt, France.
}

Data fusion is usually done using a Kalman Filter (KF). The estimated states are computed along with a covariance matrix that encodes the inherent uncertainty of the estimates. This matrix statistically evaluates the estimation error.

The accepted risk level can be defined by the Target Integrity Risk (TIR), which depends on the navigation context and any applied safety policy. The Protection Level $(P L)$ is defined as the statistical bound on the Position Error $(P E)$ such that the probability of the absolute error exceeding the $P L$ (empirical integrity risk denoted $I R$ ) is smaller or equal to the $T I R$. Therefore, for a chosen $T I R$, the system meets the integrity requirement if the $I R$ is lower than the $T I R$ :

$$
\operatorname{Pr}(P E>P L) \leq T I R
$$

The system is unavailable if $P L>A L$, where $A L$ is the Alarm Limit defined as the largest error allowable for safe operation without issuing an alert. The different integrity concepts are often shown using a Stanford-ESA diagram. This plots $P E$ against $P L$ for a set of measurements with a given $T I R$, which is helpful in assessing the performance and usability of a localization system [8].

For autonomous vehicles, it is a question of choosing the correct $T I R$ level. In the ESCAPE project ${ }^{1}$, the integrity levels for Galileo-based GNSS hybrid localization systems were estimated. The $T I R$ chosen was $10^{-6}$ per estimated location. This is for an accident probability of about $10^{-9}$. Similar figures were chosen in the SaPPART COST Action ${ }^{2}$. The hypothesis applied was that no accident should occur due to localization errors.

In practice, it is often observed that the uncertainty estimates computed from the Kalman filter are suboptimal and not consistent. That is, PLs computed from the covariance matrix do not satisfy equation (1). This is because one or more fundamental hypotheses of the Kalman filter framework are violated. The sources of errors include linear approximations, non-Gaussian noise, non-centered noise, correlated errors, outliers, etc.

The present paper proposes a novel localization solution from an integrity perspective, and the bounding of the estimation error. Our proposed approach combines measurement rejection and position error characterization. A Fault Detection and Exclusion (FDE) step is first applied, then a $P L$ is

\footnotetext{
${ }^{1}$ http://www.gnss-escape.eu/

${ }^{2}$ http://www.sappart.net/
} 
computed using a fine-tuned Student's $t$-distribution optimized from recorded data.

The main contributions of the paper are i) using a bank of information filters for the data fusion, with an FDE that is able to handle different sensors and residuals in the state space, ii) including a Student's $t$-distribution for $P L$ computation, chosen according to a learning strategy, iii) combining measurement rejection and position error characterization in an integrity framework without the assumption of one fault at a time.

The paper is organized as follows. Section II presents a state-of-the-art of integrity applied to localization systems. The rationale behind the proposed approach is presented in section III. The multi-sensor data fusion strategy is described in section IV. The integrity study, detailed in Section V, includes the FDE step and the computation of the $P L$. The approach is tested using data acquired in public roads. The results are included and analyzed in Section VI.

\section{RELATED WORK}

Integrity determines the level of trust that can be placed in localization estimates. This can be expressed in terms of PLs defined by the acceptable integrity risk, as in equation (1). Most studies on integrity focus on the aerospace field. This section looks into the different methods that have been applied to determine levels of integrity. Three types of methods are considered: the rejection of measurements (that is, FDE), error characterization according to the source of error, and mapaided integrity methods applied to road vehicles [9], [10].

The main drawback of the FDE integrity approach when applied to intelligent vehicles is that more than one measurement may be faulty at the same time, and may therefore fail to provide a navigation solution with an associated $P L$. Error characterization approaches consist in modeling the measurement error distribution and computing a $P L$ without an FDE step. They can be applied either in the range measurement domain [11] or in the position domain [12]. Error characterization approaches can lead to large PLs in the presence of faulty measurements, increasing the unavailability of the localization system.

\section{A. Classical integrity methods and their limitations for intel- ligent vehicles}

In aviation, $P L$ s are measurement-independent. They depend on satellite geometry and the prior distribution of the errors. These PLs are therefore predictable at any point on Earth [13]. A classical application of the FDE approach to the GNSS localization problem is Receiver Autonomous Integrity Monitoring (RAIM). This monitors the integrity of the position using redundant information (e.g., pseudo-distances) to detect satellite faults. Conventional RAIM relies on a one-fault-at-atime detection scheme. New RAIM methods incorporate FDE mechanisms that allow for service continuity [14]. Integrity monitoring within the RAIM can be achieved through four different methods: least squares residuals [15], parity space [16], range comparison [17], and maximum solution separation [18].
The best-known method for $P L$ computation is the FDE slope-based method [19]. The test statistic, constructed in the observation space, follows a central Chi-squared distribution in the non-faulty case and a non-central Chi-squared in the faulty case. It assumes a single biased pseudo-range at a time. The threshold is chosen such that a given false alarm probability $\operatorname{Pr}_{f a}$ is guaranteed. The $P L$ is obtained by computing the effect of the minimum detectable bias on the horizontal $P E$.

Another method is Maximum Solution Separation (MSS). This requires a rejection step [18], [20]. MSS is based on the construction of a test statistic in the position domain. It also assumes one fault at a time.

Expecting one fault at a time is reasonable for aviation applications, but not for ground vehicles [21]. Where there are two faulty satellites simultaneously, computing the $P L$ requires examining every pair of satellites, which for $N$ satellites gives $\frac{N !}{N-2) ! \times 2 !}$ combinations to be tested [22]. When applied to GNSS constellations (e.g. GPS, Galileo, Glonass, etc.), this can become computation-intensive and thus difficult to use.

In [23], the $P L$ computation is done using the error characterization of two parameters: the User Equivalent Range Error (UERE) and the Dilution Of Precision (DOP). The UERE calculates the quality of the range measurements based on ephemeris uncertainties, receiver noise, propagation errors, and clock and timing errors. It is modeled as a Gaussian random variable with a zero mean and a standard deviation $\sigma_{U E R E}$. The DOP represents errors that are due to the satellite spatial geometric distribution. The assumption of a zero mean Gaussian distribution for GNSS errors, even when operating with differential corrections in urban environments, corresponds only rarely to reality, due to the presence of multipath and biases. The same applies to Satellite-Based Augmentation Systems (SBAS) like EGNOS [24] [25] [26].

The PLs obtained in aviation applications are too conservative for vehicular applications [9]. Airplanes travel in 14-kilometer-wide air corridors [27]. Simulations shows that while RAIM availability is around $100 \%$ in open sky, it decreases dramatically in urban environments because of the occlusion of satellite signals by high-rise buildings, even with a 100-meter-wide alert limit [28]. RAIM availability is $55 \%$ in urban environments when using the GPS-Galileo dual constellation and with $\operatorname{Pr}_{f a}=5 \times 10^{-3}$ and $P r_{m d}=5 \times 10^{-5}$. With the GPS constellation alone, this availability decreases to $7 \%$ [29].

Localization systems used for intelligent vehicles are enhanced by incorporating other proprioceptive and exteroceptive sensors like video cameras or LiDARs. In urban conditions, measurements are affected by unpredictable errors, and so the computation of the $P L$ must include the residuals on the actual measurements.

Given these limitations, new methods for integrity monitoring in road conditions are needed. The objective is to address the presence of multiple faults simultaneously, the availability of exteroceptive sensors other than GNSS, and the use of maps that store landmarks and features that can be used for localization. Attention should also be paid to non-Gaussian distributions of the errors. 


\section{B. Integrity applied to road vehicles}

Different integrity estimation methods applicable to urban environments have been proposed. In [30]-[32], the idea is to characterize and predict the degradation of the GNSS signals by implementing a collaborative spatio/temporal approach between multiple vehicles within a Vehicular Ad-hoc Network (VANET). The method estimates an effective $\sigma_{U E R E}$ using the covariance of the residual vector on a specific position. It does not assume a generic model, and includes the effects of noise and multipath on the quality of pseudo-range measurements for a specific position. However, it requires multiple vehicles, or multiple runs in the same place.

Another approach characterizes errors in the position domain rather than modeling individual pseudo-range measurement errors [12]. The Horizontal Position Error (HPE) is computed from experimental data with respect to a ground truth. The HPE is fitted by a Rayleigh distribution in open sky, using an empirical Probability Density Function (PDF) and a Cumulative Density Function (CDF). In urban environments, the pseudo-range errors are likely to be non-Gaussian due to multipath. This implies that errors generate distributions with heavy tails. To provide flexibility, the authors chose the generalized Pareto distribution. Once the CDF of the HPE is determined, the $P L$ can be obtained from the inverse of the $\mathrm{CDF}$ for a chosen missed detection probability.

The characterization of the position errors is also proposed in [33], [34]. The method is known as the Kalman Integrated Protection Level (KIPL). It is an extension of an earlier method, namely the Isotropy-Based Protection Level (IBPL) [35], [36]. The KIPL uses a KF for state estimation and error propagation. To calculate the $P L$, errors are assumed to be distributed according to a multivariate Student's $t$ distribution. The total error of the KF is considered to be the sum of contributions from several error sources. For each measurement $i$, the position error is separated into two distinct elements: an error of the measurement $i$ projected to the state model, and an error of the measurement $i$ accumulated over previous epochs. Given that the sum of two $t$-distributions is not $t$-distributed, the authors use a fitting method to adjust the sum to a $t$-distribution. Once the distribution is known, the associated protection level $P L_{i}$ can be computed and the $P L$ is considered to be the sum of $P L_{i}$. To derive the KIPL, the authors make certain assumptions, such as uncorrelated Kalman gain and a diagonal innovation covariance matrix.

The various methods mentioned above characterize errors without removing large biases, that is to say no FDE is applied. These biases may increase the unavailability of the system.

For the sake of completeness, we also need to mention emergent map-aided integrity methods. Empirical methods that use map-matching to estimate integrity are proposed in [37]. The integrity of the map matching is considered as high if the uncertainty associated with the position solution is small, and if the absolute differences between the vehicle pose and the road link (in the map) in terms of distance and heading are small. For this purpose, a fuzzy logic model is used to derive a metric between 0 and 100 that reflects the map matching confidence.
[38] used precise point-positioning GNSS and a monocular camera together with an HD map to study the precision and reliability of the vehicle state estimation in the presence of errors. This approach examined internal reliability (FDE) and external reliability (the impact of undetected faults).

Another approach addresses lane level integrity [39]. GNSS and vehicle odometry are merged with a digital map through a Particle Filter (PF). The integrity of the map-matched position is represented by two variables. The Lane Occupancy Probability $(\mu L O)$ of a segment is obtained by adding the weights of the particles corresponding to that segment. The confidence level of positioning and map matching is estimated. This approach also estimates the Lane Positioning Protection Level $(L P P L)$ defined by $k \sigma_{\text {pos }}$, where $k$ is calculated using a Rayleigh distribution according to a missed detection probability and $\sigma_{p o s}$ obtained from the maximum eigenvalue of the covariance matrix along the $x$ and $y$-coordinates. The position is valid if $\mu L O>\mu L O_{\text {threshold }}$ and $L P P L<L P P L_{\text {threshold }}$.

Integrity monitoring can also be derived using a $\mathrm{PF}$ that estimates a set of likely map-matched hypotheses, according to the $P L$ provided by a GNSS receiver [40]. That is, the current fix of the GNSS receiver is used to select the coherent hypotheses using a Mahalanobis distance. Where the remaining set contains a single hypothesis (as opposed to multiple hypotheses or no hypotheses at all), it may be decided to use the position estimate.

\section{PROPOSED APPROACH}

It is our assertion in this paper that localization systems for intelligent vehicles need to use redundant and complementary technologies. GNSS systems are indispensable, given their Earth-wide coverage, their constantly improving positioning quality and the low cost of receivers. These systems are hybridized using inertial systems or Dead-Reckoning (DR) using wheel speed measurements, for instance. HD maps are a valuable complementary source of positioning information. By combining the map with measurements from exteroceptive sensors such as LiDARs and cameras, the car can derive very accurate localization information. This paper formulates an integrity concept applicable to any multi-sensor localization system.

The approach addresses the constraints encountered in land navigation, such as errors due to multipath and NLOS of GNSS satellites [41], [42], measurement errors of the exteroceptive sensors, landmarks and features errors in the map, and data association issues.

Our proposed approach combines measurement rejection and position error characterization. It merges the information provided by all information sources. The data fusion of a DR system with a low-cost single-frequency GNSS receiver is considered. A smart camera is used to provide measurements of detected lane markings georeferenced in an HD map (see Figure 1).

Measurements are affected by errors mainly due to the navigation environment, although errors on the camera measurements may originate in the measurement itself or in the 


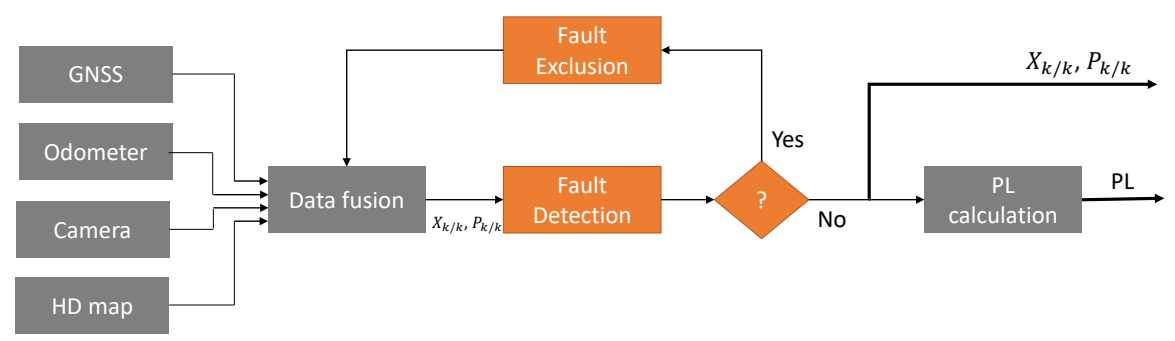

Figure 1. General architecture applied to the problem addressed in this paper. The fusion process takes as input information from GNSS, odometer, camera and HD map. The system performs an FDE step before providing pose estimates $X_{k / k}$ along with covariance matrices $P_{k / k}$ and $P L s$.

map. These errors have a direct effect on the integrity of the localization if they are not detected and excluded from the fusion procedure. Therefore, an FDE step is added in order to test the consistency of the exteroceptive measurements with the DR measurements. Our approach is based on the use of the Information Filter (IF) for state estimation, and Mahalanobis distance in the state space for fault detection. The IF is mainly advantageous for multi-sensor data fusion and for FDE as a result of the summation part that appears in the update step [43].

Once all the detected faulty measurements are removed, an error model is built to compute a consistent $P L$. For this step, we use a Student's $t$-distribution. This is characterized by a degree of freedom (dof) that shapes the tail of the distribution, leading to a more consistent $P L$ for small integrity risk (e.g., $10^{-3}$ ). The heavy tail of the Student's $t$-distribution handles large errors with low occurrence probabilities, and this cannot be done when using a Gaussian distribution. The tuning of the dof can be done experimentally in a learning step according to the navigation environment (urban, suburban, open sky, etc.). This is in line with the strategy for deploying initially autonomous vehicles within a well-defined operational design domain at first. For intelligent vehicles, $P L s$ are expressed in the Along Track (AT) and Cross Track (CT) directions that describe the relative position of the vehicle with respect to the road. This allows different alarm thresholds depending on the direction.

\section{Multi-SENSOR DATA FUSION}

At instant $k$, the state vector is considered to be the position and the heading of the vehicle in an East-North-Up (ENU) frame denoted $R_{O}$ :

$$
X=\left[\begin{array}{lll}
x & y & \theta
\end{array}\right]^{T}
$$

The propagation equation is obtained using DR as follows:

$$
\begin{aligned}
X_{k+1 / k} & =f\left(X_{k / k}, u_{k}\right)+v_{k} \\
& =\left[\begin{array}{c}
x_{k / k}+\cos \left(\theta_{k / k}+\frac{\omega_{k}}{2}\right) \Delta_{k} \\
y_{k / k}+\sin \left(\theta_{k / k}+\frac{\omega_{k}}{2}\right) \Delta_{k} \\
\theta_{k / k}+\omega_{k}
\end{array}\right]+v_{k}
\end{aligned}
$$

where $u_{k}=\left[\begin{array}{ll}\Delta_{k} & \omega_{k}\end{array}\right]^{T}$ is the input vector composed of an elementary rotation $\omega_{k}$ obtained from the gyro and an elementary displacement $\Delta_{k}$ obtained from the wheel speed sensors, and $v_{k}$ is modeled as Gaussian white noise with covariance matrix $Q_{k}$.

The information matrix, defined as the inverse of the covariance matrix, is written as:

$$
Y_{k+1 / k}=P_{k+1 / k}^{-1}=\left(F_{k} P_{k / k} F_{k}^{T}+B_{k}\left(Q_{u}\right)_{k} B_{k}^{T}+Q_{k}\right)^{-1}
$$

where

$Q_{u} \quad$ is the covariance matrix corresponding to the input vector;

$F_{k} \quad$ and $B_{k}$ are the Jacobian matrices computed as $F_{k}=$ $\left.\frac{\partial f}{\partial x}\right|_{X=X_{k / k}}$ and $B_{k}=\left.\frac{\partial f}{\partial u}\right|_{u=u_{k}}$.

The information vector is obtained from the state vector and takes the form:

$$
y_{k+1 / k}=Y_{k+1 / k} X_{k+1 / k}
$$

The information matrix and information vector are updated according to equations (6) and (7):

$$
\begin{aligned}
& Y_{k / k}=Y_{k / k-1}+\sum_{i=1}^{n} I_{i, k} \\
& y_{k / k}=y_{k / k-1}+\sum_{i=1}^{n} i_{i, k}
\end{aligned}
$$

where $n$ is the number of observations and $I_{i, k}$ and $i_{i, k}$ are the information contributions associated with the observation $Z_{i}$. The computation of these values is detailed in the following. Please note that the IF is mathematically equivalent to the KF. However, the main advantage of IF over KF can be seen in equations (6) and (7), where the update step is modeled as a simple summation. This facilitates the FDE step.

The observations from the GNSS receiver and the smart camera are merged with the DR measurements to improve the localization so as to reach a lane-level accuracy.

The fusion of DR with the GNSS is done through a loosely coupled architecture. Therefore, the observation vector corresponding to the GNSS measurement is written as:

$$
Z_{\mathrm{GNSS}}=\left[\begin{array}{l}
x_{\mathrm{GNSS}} \\
y_{\mathrm{GNSS}}
\end{array}\right]=\left[\begin{array}{c}
t_{x} \cos \theta-t_{y} \sin \theta+x \\
t_{x} \sin \theta+t_{y} \cos \theta+y
\end{array}\right],
$$

where $t_{x}$ and $t_{y}$ are the translation of the antenna (frame $R_{G}$ ) with respect to the body frame $\left(R_{B}\right)$ located at the middle of the rear wheel axis of the vehicle where the pose $(x, y, \theta)$ is defined (Figure 2). 


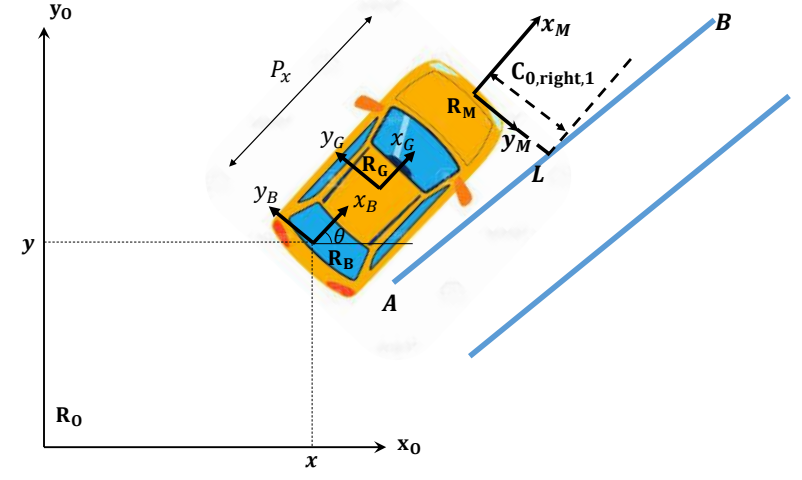

Figure 2. Frames representation. The vehicle pose $\left[\begin{array}{ccc}x & y & \theta\end{array}\right]^{T}$ is expressed in a global ENU frame $R_{O}$ and is attached to the body frame $R_{B}$ situated at the middle of the rear wheel axis. GNSS measurements are given in the antenna frame $R_{G}$. The camera measurements are expressed in the frame $R_{M}$ situated at a distance $P_{x}$ in front of $R_{B}$. The camera measures the lateral distance $C_{0}$ to the point $L$ in a road marking segment $[A B]$.

The GNSS information contribution is given by:

$I_{\mathrm{GNSS}, k}=H_{\mathrm{GNSS}, k}^{T} R_{\mathrm{GNSS}, k}^{-1} H_{\mathrm{GNSS}, k}$

$i_{\mathrm{GNSS}, k}=H_{\mathrm{GNSS}, k}^{T} R_{\mathrm{GNSS}, k}^{-1}\left[\left(Z_{\mathrm{GNSS}, k}-\hat{Z}_{\mathrm{GNSS}}\right)+H_{\mathrm{GNSS}, k} X_{k / k-1}\right]$

where $H_{\mathrm{GNSS}}$ is the observation matrix:

$$
H_{\mathrm{GNSS}}=\left[\begin{array}{rrr}
1 & 0 & -t_{x} \sin \theta_{k / k-1}-t_{y} \cos \theta_{k / k-1} \\
0 & 1 & t_{x} \cos \theta_{k / k-1}-t_{y} \sin \theta_{k / k-1}
\end{array}\right]
$$

and $R_{\mathrm{GNSS}}$ is the covariance matrix of the observation noise.

The smart camera detects lane markings and returns the coefficients of a Taylor expansion of a clothoid in a virtual frame $R_{M}$ located at the center of the front bumper, while the camera itself is located behind the windscreen:

$$
y=C_{3} x^{3}+C_{2} x^{2}+C_{1} x+C_{0}
$$

The camera is able to detect up to four markings at a time, two on each side: right and left. In this paper, we consider only the use of the parameter $C_{0}$ (the lateral distance between the lane marking and the frame $R_{M}$ ):

$$
Z_{i}=C_{0}
$$

where $i$ can represent $C_{0, \text { left, } 1}, C_{0, \text { left, } 2}, C_{0, \text { right, } 1}, C_{0, \text { right, } 2}$ if they exist.

The camera observation model developed in [7] can be written as:

$$
C_{0}=\frac{\left(P_{x} \sin \theta+y-y_{A}\right) \cdot x_{A B}-\left(P_{x} \cos \theta+x-x_{A}\right) \cdot y_{A B}}{x_{A B} \cdot \cos \theta+y_{A B} \cdot \sin \theta}
$$

where the lane marking corresponding to the map-matched segment $[A B]$ at coordinates $\left[x_{L}, y_{L}\right]$ is expressed in frame $R_{O}$ as:

$$
{ }^{o} L={ }^{o} T_{B} \cdot{ }^{B} T_{M} \cdot{ }^{M} L
$$

with ${ }^{O} T_{B}$ and ${ }^{B} T_{M}$ the transformation matrices from $R_{B}$ to $R_{O}$ and from $R_{M}$ to $R_{B}$, respectively. Therefore,

$$
\left[\begin{array}{l}
x_{L} \\
y_{L}
\end{array}\right]=\left[\begin{array}{c}
P_{x} \cos \theta+C_{0} \sin \theta+x \\
P_{x} \sin \theta-C_{0} \cos \theta+y
\end{array}\right]
$$

where ${ }^{M} L=\left[0, C_{0}\right]$ and $P_{x}$ is the distance between $R_{B}$ and $R_{M}$ (Figure 2).

\section{INTEGRITY PROCESSES}

\section{A. Fault detection and exclusion}

The observations from the GNSS receiver or from the camera can be faulty (or erroneous). The origin of the fault can come from the sensor, the map or the environmental conditions. Erroneous measurements (faults), if not detected, directly affect the localization quality. Hence, an FDE step is added, where the Mahalanobis distance between the predicted state and the updated state is used as a residual:

$$
r_{k}=\left(X_{k / k}-X_{k / k-1}\right)^{T} Y_{k / k}\left(X_{k / k}-X_{k / k-1}\right)
$$

$X_{k / k}$ and $Y_{k / k}$ are obtained from a principal filter that uses all available measurements at instant $k$ (cf. Figure 3). Note that this residual acts in the state space and detects faults which have a direct influence on the position estimate. Faulty measurements that have a negligible effect on position are not excluded from the fusion procedure. In some cases, using measurements with errors can be preferable to isolating them [44].

If $r_{k}$ exceeds a threshold, a fault is detected and its origin has to be determined. The threshold is obtained from a Chisquared distribution with 3-degrees of freedom according to a given false alarm probability $\left(\operatorname{Pr}_{f a}=0.05\right.$ in this paper).

For the exclusion step, a set of residuals $r_{i}$ is generated using a bank of information filters $E I F_{i}$ :

$$
r_{i, k}=\left(X_{k / k, i}-X_{k / k-1}\right)^{T} Y_{k / k, i}\left(X_{k / k, i}-X_{k / k-1}\right)
$$

where $X_{k / k, i}$ and $Y_{k / k, i}$ are obtained from a filter $\left(E I F_{i}\right)$ that uses only the observation $i$ in the update step $\left(i \in\left\{\mathrm{GNSS}, C_{0 \text {,left, } 1}\right.\right.$, $\left.\left.C_{0, \text { left }, 2}, C_{0, \text { right }, 1}, C_{0, \text { right }, 2}\right\}\right)$. If $r_{i, k}$ exceeds the threshold value, the measurement $i$ is excluded from the fusion procedure by simply subtracting its information contribution from the principal filter (Eq. (6) and (7)). For $r_{i, k}$ the threshold is also obtained from a Chi-squared distribution with 3-degrees of freedom. In this paper, for simplification, the false alarm probability is chosen to be the same for the different residuals. In practice, a threshold optimization has to be done to improve the detection quality.

At a given time the complete set of residuals is not always available, since the sensor measurements are managed asynchronously, but this is not an issue for the method. For instance, the availability of the camera measurements depends on the navigation environment.

Using this approach, a distinction can be made between camera faults (including map-matched errors) and map faults. Map faults refer to errors in the HD map. As the camera gives an indication of the quality of the lane marking detection, a lane marking with a quality below a given value is 


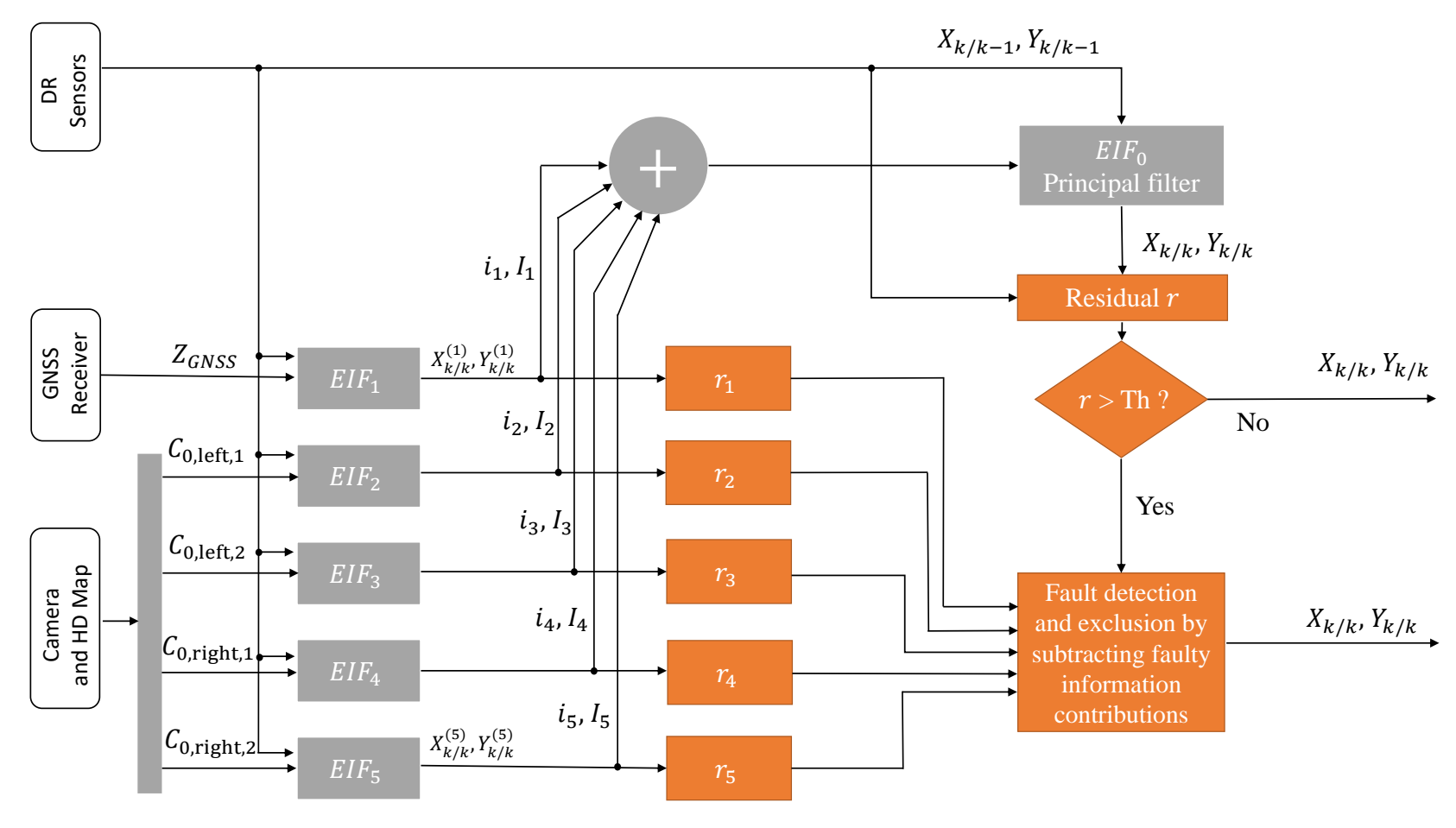

Figure 3. Multi-sensor fusion with FDE. The filters use the camera measurements and the HD map as input.

rejected. This initial filtering considerably reduces the number of outliers. Given that the camera is able to detect up to four lane markings, two on each side, the redundancy can be used to differentiate between camera and map faults. Let us take the following example for illustration. At instant $k$, suppose the camera observes simultaneously two lane markings on the left (and so provides two measurements $C_{0, \text { left, } 1}$ and $C_{0, \text { left }, 2}$ ). If the residual $r_{C_{0} \text {, left, } 1}$ exceeds the threshold value, we can conclude that something is wrong with this measurement. At this stage, we cannot specify if the error comes from the camera or from the map. Suppose now that the residual $r_{C_{0} \text { left, } 2}$ is below the threshold. In this case, at the same instant and on the same side, the camera has one good match and one bad. In these conditions, it is likely that the error comes from the map, since the two measurements are provided with high confidence. This is a phenomenon that we have observed several times by comparing the results using the HD map. A flag can be stored in the map to indicate that the map-matched segment is potentially erroneous. Now, if $r_{C_{0}, \text { left, } 2}$ is greater than the threshold, it cannot be concluded either that there is or that there is not an error in the map. Indeed, if the two observations on the same side are faulty, the error may be due to the environment, the camera itself, or the map-matching procedure. Likewise, if only one measurement is available on a given side, it is not possible to differentiate between camera faults and map faults. Finally, if all the residuals $r_{i}$ indicate the presence of errors, the hypothesis of a filtering problem or DR problem is likely and an alarm has to be raised. The origin of the error (camera or map) does not affect the exclusion procedure. If $r_{C_{0}, j}$ indicates the presence of a fault, the measurement $C_{0, j}$ is not used in the update step,

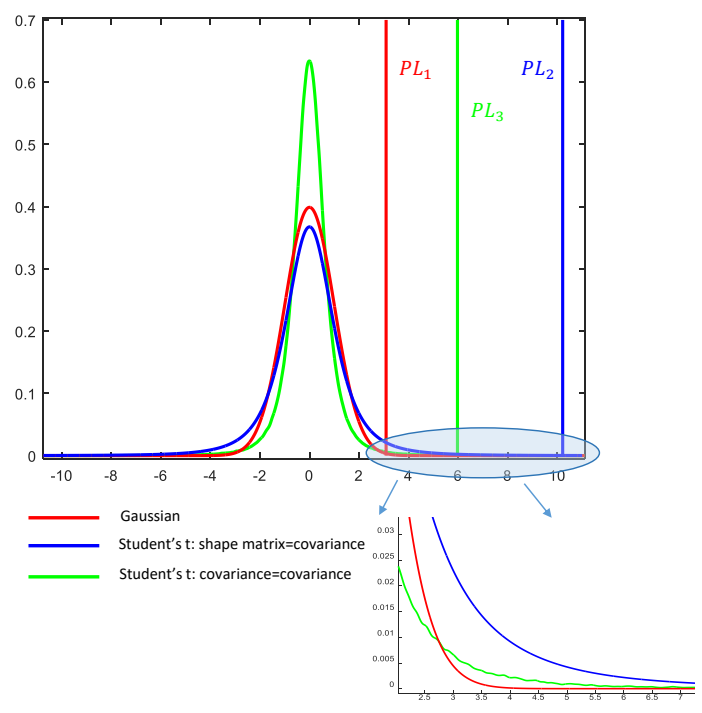

Figure 4. Comparison of two Student's $t$-distribution choices (same degree of freedom): in red: Gaussian distribution and the associated $P L_{1}$, in blue: Student's $t$-distribution with shape matrix equal to the covariance of the Gaussian and the associated $P L_{2}$, in green: Student's $t$ - distribution with covariance matrix equal to the covariance of the Gaussian and the associated $P L_{3}$.

irrespective of the origin of the measurement error.

\section{B. Protection level computation}

After having removed the detected faulty measurements, the objective is now to bound the estimation errors. The 
aim is to avoid an underestimation of the $P L$ that could be due to undetected biases, non-Gaussian error distributions, or linearization of non-linear models that could result in an underestimation of the covariance matrix. Hence, when computing the $P L$, the multivariate Gaussian distribution is replaced by a multivariate Student's $t$-distribution, which has a heavier tail than the Gaussian distribution and is able to handle errors with small probabilities that are not taken into account when using the Gaussian distribution [45].

A random variable $X$ is said to follow a multivariate $t$ distribution with mean vector $\mu$, shape matrix $R_{S}$ and dof $v$, if it can be written in the form [46]:

$$
X=\mu+\omega^{-\frac{1}{2}} y
$$

where $\omega$ is distributed according to a Gamma distribution $\Gamma\left(\frac{v}{2}, \frac{v}{2}\right)$ and $y$ has a zero-mean Gaussian distribution with covariance $P$. It is denoted as $X \sim S t\left(\mu, R_{S}, v\right)$.

The dof of the Student's $t$-distribution has a direct effect on the tails of the distribution and if $v \rightarrow \infty$ the $t$-distribution converges to a Gaussian one. The covariance of the $t$-distribution is defined only if $v>2$, and in this case it can be written as $P=\frac{v}{v-2} R_{S}$.

Figure 4 shows, for a given target integrity risk $(T I R=$ $10^{-3}$ ), the interest of a $t$-distribution for $P L$ computation as compared to a Gaussian distribution. The blue plot represents a $t$-distribution with a shape matrix equal to the covariance of the Gaussian distribution. Here the $P L$ obtained from this $t$-distribution is very conservative in comparison to that obtained from the Gaussian. The green plot represents a $t$ distribution with covariance equal to the covariance of the Gaussian distribution. For small integrity risk, the $P L$ obtained from this $t$-distribution is more conservative than that obtained using the Gaussian distribution, but it is less conservative than that illustrated in blue. For intelligent vehicles, the aim is to avoid an undervaluation of the $P L$ under small integrity risks, without however being too conservative; otherwise the system will become unavailable. For this purpose, the matrix $P_{k / k}$ will be considered as the covariance matrix of a multivariate Student's $t$-distribution with a fixed dof $v$.

The horizontal protection level is expressed as follows [33]:

$$
P L_{k}(\alpha)=K(\alpha, v) \sqrt{v \times \max \left(\text { eigenvalue }\left(R_{S}\right)\right)}
$$

where $R_{S}$ is the shape matrix of the $t$-distribution with a dof $v$. Given that the covariance can be written as $P_{k / k}=\frac{v}{v-2} R_{S}$, the $P L$ can be expressed as:

$$
P L_{k}(\alpha)=K(\alpha, v) \sqrt{v-2} \sqrt{\max \left(\operatorname{eigenvalue}\left(P_{k / k}\right)\right)},
$$

where $K$ is obtained from a multivariate $t$-distribution with dof $v$ and according to a given confidence level $\alpha$. The $K(\alpha, v)$ value can be computed by numerically solving the following equation [33]:

$$
\alpha=\frac{2}{B\left(\frac{d}{2}, \frac{v}{2}\right)} \int_{K}^{\infty} \frac{y^{d-1}}{\left(1+y^{2}\right)^{\frac{v+d}{2}}} d y .
$$

where $B\left(\frac{d}{2}, \frac{v}{2}\right)$ is the Beta function and $d$ is the dimension of the $t$-distribution; for horizontal $P L, d=2$.
For intelligent vehicles, we are interested in PLs in the AT and CT directions. Therefore, the covariance matrix in the ENU frame is projected to the (AT, CT) frame.

Let $\left(\lambda_{1}, \lambda_{2}\right)=$ eigenvalue $\left(P_{k / k(1,2),(1,2)}\right)$ and $\left(V_{1}, V_{2}\right)=$ eigenvector $\left(P_{k / k(1,2),(1,2)}\right)$ be the eigen values and eigen vectors of the $2 \times 2$ position component of the covariance matrix $P_{k / k}$. The expression of $V_{1}$ and $V_{2}$ in the (AT, CT) frame is given as:

$$
\left[\begin{array}{l}
V_{1, A T} \\
V_{1, C T}
\end{array}\right]=R_{\text {proj }} \cdot V_{1} \text { and }\left[\begin{array}{l}
V_{2, A T} \\
V_{2, C T}
\end{array}\right]=R_{\text {proj }} \cdot V_{2}
$$

where

$$
R_{\text {proj }}=\left[\begin{array}{cc}
\cos \theta & \sin \theta \\
-\sin \theta & \cos \theta
\end{array}\right]
$$

with $\theta$ the orientation of the vehicle.

The AT and CT PLs are obtained as:

$$
\begin{aligned}
& P L_{A T}=K(\alpha, v) \sqrt{v-2} \sqrt{\max \left(\left|\lambda_{1} V_{1, A T}\right|,\left|\lambda_{2} V_{2, A T}\right|\right)} \\
& P L_{C T}=K(\alpha, v) \sqrt{v-2} \sqrt{\max \left(\left|\lambda_{1} V_{1, C T}\right|,\left|\lambda_{2} V_{2, C T}\right|\right)}
\end{aligned}
$$

The errors in the AT and CT directions are expressed in terms of the error in the East $\left(\mathrm{er}_{x}\right)$ and North $\left(\mathrm{er}_{y}\right)$ directions as:

$$
\left[\begin{array}{l}
\mathrm{er}_{A T} \\
\mathrm{er}_{C T}
\end{array}\right]=R_{p r o j}\left[\begin{array}{l}
\mathrm{er}_{x} \\
\mathrm{er}_{y}
\end{array}\right]
$$

The dof of the Student's $t$-distribution is defined based on a learning strategy which includes prior knowledge of the driving context. Further details are provided in the next section.

\section{EXPERIMENTAL STUDY}

Experiments were performed in the city of Rambouillet (figure 5). The test vehicle is equipped with a Ublox 8T single frequency multi-constellation GNSS receiver and a production camera for lane detection (Mobileye EQ3). An HD-map of the area, designed for testing autonomous vehicles, is also available. The location ground truth is generated using a high-end system (NovAtel SPAN-CPT) with RTK corrections. Acquisitions were made at $50 \mathrm{~Hz}$ for the ground truth, $50 \mathrm{~Hz}$ for the dead-reckoning, $2 \mathrm{~Hz}$ for the GNSS fix, and $35 \mathrm{~Hz}$ for the camera (sub-sampled at $3.5 \mathrm{~Hz}$ to reduce time correlation errors).

Three trajectories with several runs were carried out and separated into training and testing trajectories. Each of the three trajectories was repeated twice, the first run for the learning phase (training trajectory) and the second for the test phase (test trajectory). Trajectory \#1 is about $4.2 \mathrm{Km}$ long with U-turns forming a loop. Trajectory \#2 is about $2.4 \mathrm{Km}$ long, and includes several roundabouts with U-turns forming a loop. These first two trajectories are shown respectively in blue and yellow in the left panel of Figure 6. Trajectory \#3 is about $3.2 \mathrm{Km}$ long, as shown in the right panel of Figure 6. The vehicle was driven along the selected trajectories for the 


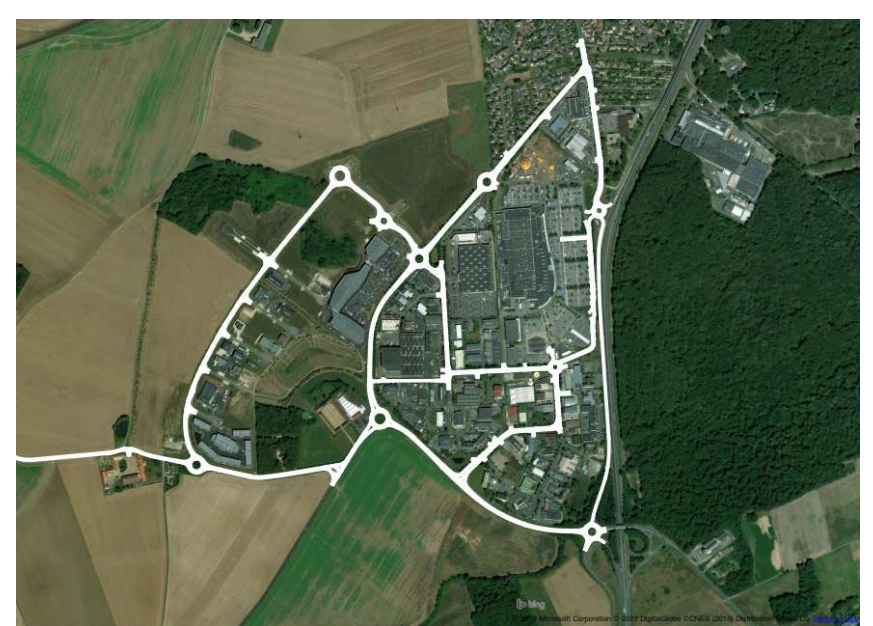

Figure 5. Display of the available HD map (in white) in the city of Rambouillet, France.

learning phase and to perform the tests. The vehicle speed was between 0 and $60 \mathrm{~km} / \mathrm{h}$ with a mean value around $30 \mathrm{~km} / \mathrm{h}$.

Regarding the environment, the Rambouillet area comprises tree canopies and surrounding vegetation. Dense trees and vegetation are present on many parts of trajectories \#2 and \#3. Buildings are present on some parts of trajectory \#1 while other parts can be considered as open sky. For the purposes of this paper, the three trajectories will be considered as belonging to the same environment (sub-urban), since a finer classification is difficult. The HD map used has centimeter accuracy, but some markings are missing and others rendered obsolete by environment change. Regarding the quality of the lane markings, some have been painted recently, while others are older, but this is something for which we do not possess a usable classification. However, the onboard camera provides an indication of the quality of the detected lane marking. When the quality is below a threshold value, lane-marking information is discarded before the sensor fusion with FDE.
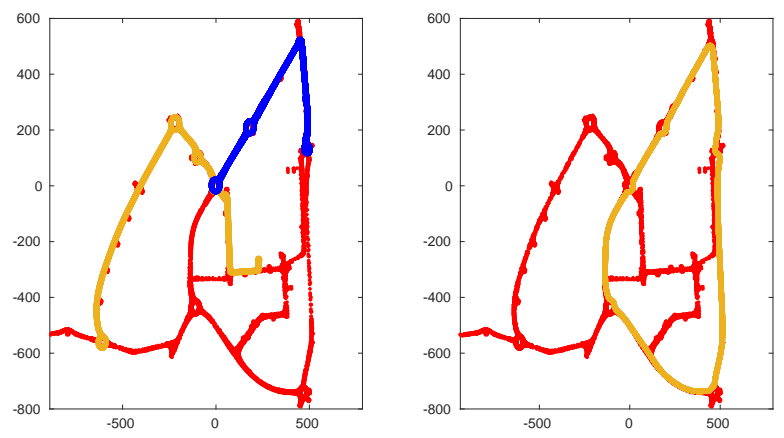

Figure 6. The three different trajectories. The red color represents the backbone of the map. Left plot: In yellow, trajectory \#1 $(\sim 4.2 \mathrm{~km})$ with a round trip. In blue, trajectory \#2 $(\sim 2.4 \mathrm{~km})$ with a round trip and a number of roundabouts. Right plot: In yellow, trajectory \#3 $(\sim 3.2 \mathrm{~km})$. On the right side of this trajectory, the lane markings are not stored in the HD map but are detected by the camera.

Below, the reporting of our experiments is separated into two subsections, dealing respectively with internal integrity and external integrity. The first of the two subsections is a validation of the multi-sensor data fusion with the FDE step. The second is the validation of the $P L$ computation based on Student's $t$-distribution. The strategy of learning the dof in the AT and CT directions is presented in detail.

\section{A. Internal integrity}

We first show experimentally the importance of having an FDE scheme in the data fusion process. Table I shows the errors before and after the FDE step for the three test trajectories. A reduction can be seen in the mean and max errors following the FDE step. For example, for the test trajectory \#1, in the AT direction, the largest error falls from $20.42 \mathrm{~m}$ before the FDE step to $1.06 \mathrm{~m}$ after the FDE step. Likewise, in the CT direction, the error falls from $19.70 \mathrm{~m}$ to $1.03 \mathrm{~m}$. The results for this trajectory are among the best results in our dataset in terms of accuracy as a result of the small GNSS errors in this experiment. Please note that for the test trajectory \#2, the FDE does not significantly improve the accuracy because there were few outliers. For the trajectory \#3, the improvement is mainly in the CT direction, where the mean error falls from $0.53 \mathrm{~m}$ before the FDE step to $0.46 \mathrm{~m}$ after the FDE step. The errors are in particular map errors detected at the beginning of the trajectory.

For a clearer illustration, the detailed results corresponding to the test trajectory \#1 are shown below.

The residual used for the fault detection with the associated threshold is shown in figure 7 . When this residual indicates the presence of faults, the set of residuals used for the fault exclusion is generated as shown in figure 8. Figure 8a shows the residual corresponding to the GNSS measurements, and figures $8 \mathrm{~b}$ and $8 \mathrm{c}$ show the residuals corresponding to the camera measurements on the left and right sides, respectively. By comparing these residuals to the threshold value, it can be seen that the errors are mostly detected on the camera measurements.

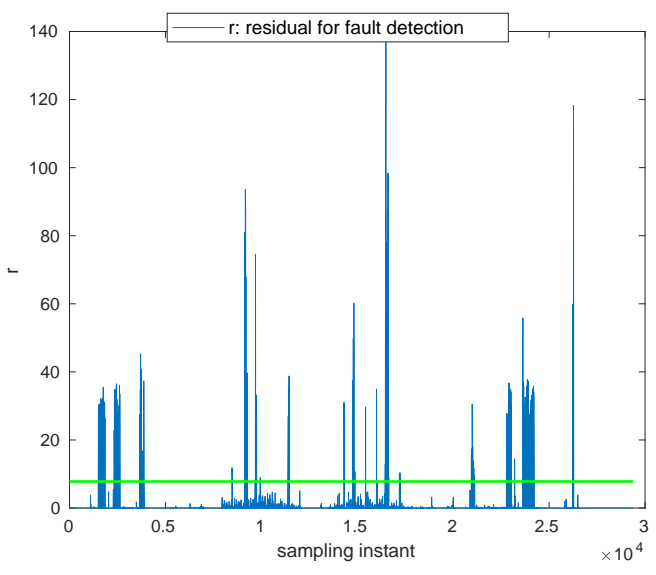

Figure 7. Residual $r$ for fault detection. In green the threshold value (test trajectory \#1). 
Table I

HORIZONTAL POSITION ERROR IN METERS BEFORE AND AFTER THE FDE STEP APPLIED TO THE TEST TRAJECTORIES.

\begin{tabular}{ccccccc}
\hline & \multicolumn{3}{c}{ After FDE } & \multicolumn{3}{c}{ Before FDE } \\
\hline Test trajectories & error & $\max \operatorname{abs}(\mathrm{AT})$ & $\max \operatorname{abs}(\mathrm{CT})$ & error & $\max \operatorname{abs}(\mathrm{AT})$ & $\max \operatorname{abs}(\mathrm{CT})$ \\
\hline Traj. \#1 $(\sim 4.2 \mathrm{~km})$ & 0.45 & 1.06 & 1.03 & 0.80 & 20.42 & 19.7 \\
\hline Traj. \#2 $(\sim 2.4 \mathrm{~km})$ & 2.48 & 3.28 & 2.93 & 2.48 & 3.29 & 2.93 \\
\hline Traj. \#3 $(\sim 3.2 \mathrm{~km})$ & 2.11 & 3.02 & 2.97 & 2.11 & 3.42 & 2.95 \\
\hline
\end{tabular}

The differentiation between camera faults (including mapmatching) and map faults is shown in figure 9. Blue indicates that the error comes from the map, while red indicates that the origin of the error cannot be specified, for one of the two reasons presented in section $\mathrm{V}$-A. Looking, for example, at figure $8 \mathrm{~b}$, it will be remarked that around the sampling instant 9693 (highlighted zone), $r_{\text {cameraL2 }}$ indicates the presence of faults while $r_{\text {cameraL1 }}$ is error free. Therefore, at these sampling instants, the faults are deemed to come from the map, and this is represented in figure $9 \mathrm{a}$ by the blue lines around the sampling instant 9693. In the same way, around the sampling instant 1522 (highlighted zone), the map is deemed erroneous, since $r_{\text {cameraL1 }}$ indicates the presence of faults while $r_{\text {cameraL2 }}$ is error free. Figure 10 shows two areas where a map fault has been detected. In these areas, the camera visualizes two lane markings on the left sides (the link border and the centerline) while only the link border is present in the map. This discrepancy led to an incorrect association. Therefore, the detected map faults are actually map errors.

Figure 11 shows the errors when using only GNSS measurements in relation to the multi-sensor data fusion before and after the FDE step. Before the FDE step, the effect of the errors on the position estimates is noticeable. The surges visible before the FDE step (in green) disappear after the FDE step (in red). The camera leads to a noticeable improvement in the CT direction with respect to the GNSS-only case. In the AT direction, the data fusion (with FDE) eliminates some outliers that occur with GNSS only, but does not improve overall accuracy, since the visual features do not provide longitudinal constraints. In some parts of the trajectory, the longitudinal error after the data fusion and after the FDE step is greater than with GNSS only, and this is mainly due to the drift of the odometry model. In other cases, some of the increases (the small surges) are due to undetected map-matching errors. This is an issue that could have been handled by optimizing the threshold, but that was not the purpose of this paper.

\section{B. External integrity}

In order to study the computation of the PLs, a TIR of $10^{-3}$ was chosen, since we did not have enough samples to test with smaller integrity risks.

The procedure for tuning the best dof value is illustrated in table II. After the FDE step, the IR was computed on the training trajectories for different values of the dof $(v=4$ to $v=100$, corresponding to a Gaussian distribution). In the CT direction, the largest value of the dof that satisfies the IR requirement overall is seen to be $v=9$ (since $0.87 \times 10^{-3}<10^{-3}$ ), although the TIR is not satisfied separately for the training trajectory \#3.

The same methodology is applied in the AT direction, where $v=5$ has to be chosen. It will be remarked that this dof is not the same as in the CT direction, given that GNSS measurements are the only exteroceptive information available in the AT direction (the camera only detects marking in the CT direction). This explains the large error in the AT direction in some parts of the trajectory, where the GNSS is sometimes biased by about $3 \mathrm{~m}$. This result would be different with a differential GNSS receiver, or if the GNSS biases were estimated in the filter. Moreover, it is clear from table II that it is unsuitable to use a Gaussian distribution for bounding the errors in this environment, since for $v=100$ the measured IRs are a long way above the specified values. To conclude, for this suburban environment and using the considered localization system, Student's $t$ - distributions with $v=9$ and $v=5$ are required to bound the errors in the $\mathrm{CT}$ and $\mathrm{AT}$ directions respectively.

Figure 12 shows the errors with the computed PLs in the AT and CT directions after the FDE step applied to the test trajectory \#1. The dof of the Student's $t$-distribution is set to $v=5$ in the AT direction and $v=9$ in the CT direction. Using these values, the computation of the PLs is consistent since the empirical IR is equal to 0 (which is smaller than $10^{-3}$ ) in both directions. When no FDE step is added, and using the same dof as before, the measured frequencies of errors that exceed the $P L$ (empirical IR) were 0.027 and 0.051 in the AT and CT directions respectively. Likewise, figure 13 shows similar results and highlights the importance of the FDE step for external integrity. Indeed, without FDE, the inconsistency of the computed $P L$ persists in presence of large biases even when small degrees of freedom are used (for $v=2.4$, the empirical IRs were 0.019 and 0.009 in the AT and CT directions respectively). In addition, it will be noted that the $P L$ decreases in the presence of undetected faults, since the erroneous measurement leads to an increase in the quantity of information, which lowers the values in the covariance matrix. Therefore, computing consistent $P L$ in the presence of faulty measurements is not possible with the presented methodology.

Let us now look at the consistency of the computed $P L$ on the different test trajectories (see Table III). In the CT direction (resp. AT direction), the Student's $t$-distribution with 


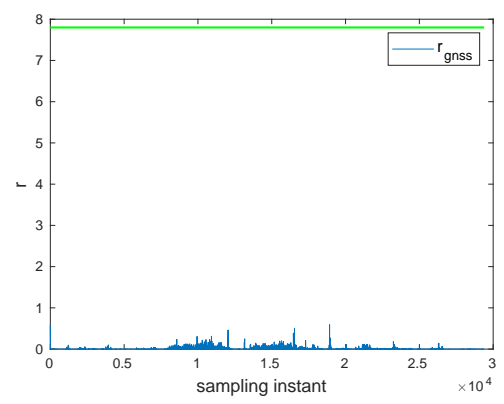

(a) $r_{G N S S}$

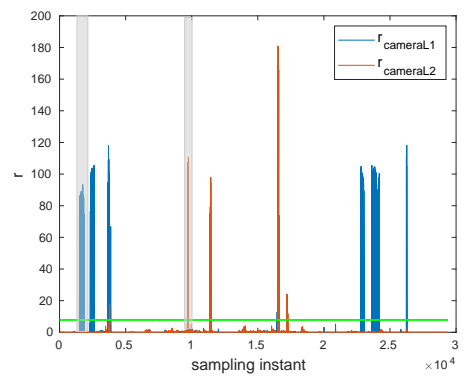

(b) $r_{\text {camera Left ,1 }}$ (blue), $r_{\text {camera Left,2 }}$ (red)

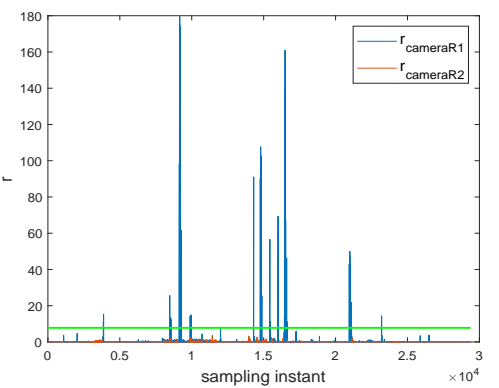

(c) $r_{\text {camera Right ,1 }}$ (blue), $r_{\text {camera Right ,2 }}$ (red)

Figure 8. Residuals $r_{i}$ for fault exclusion. In green the thresholds (test trajectory \#1).

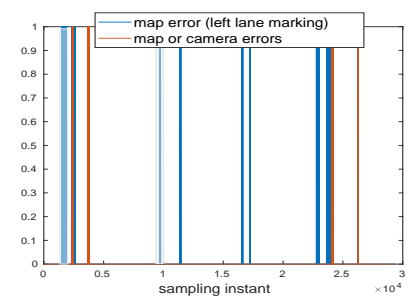

(a) Case 1: left case

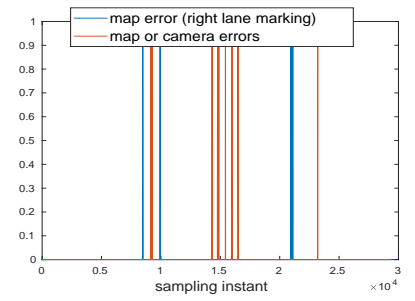

(b) Case 2: right case
Figure 9. Errors in the map (blue) and indiscernible errors between the map and the camera (red).

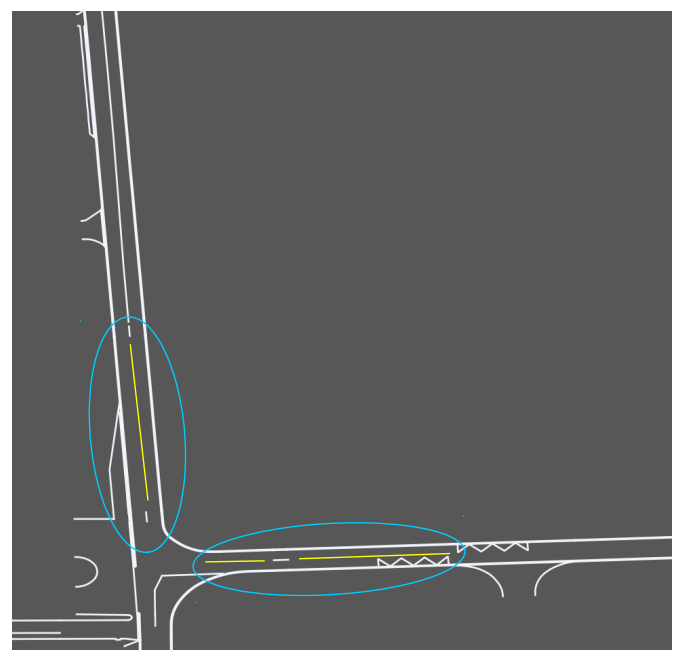

Figure 10. In blue the areas where map errors are detected, and in yellow the missing markings.

$v=9$ (resp. $v=5$ ) is suitable for $P L$ computation, since the empirical IR value is 0 in the AT direction and $0.5 \times 10^{-3}$ in the CT direction.

As an illustration of the values of the calculated $P L$ s with the chosen dof, figure 14 shows their values on the test trajectory \#2. Several rounds on the roundabouts explain the sinusoidal shape that appears in this figure. The camera cannot operate on highly curved roads, which explains the unavailability of its measurements on the roundabouts and the increase in the $P L$ value, which is around $0.97 \mathrm{~m}$ in the $\mathrm{CT}$ direction when
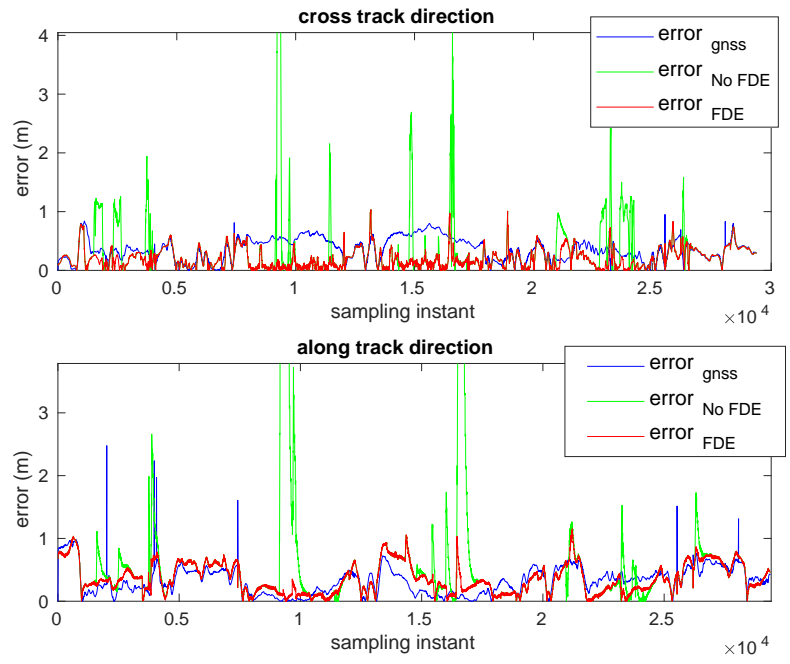

Figure 11. Errors after data fusion with FDE (in red) compared to data fusion without FDE (in green) and to GNSS (in blue). Sampling period of $0.02 \mathrm{~s}$ corresponding to the period of the DR sensors. Test trajectory \#1.

camera measurements are available, and about $3.4 \mathrm{~m}$ in the AT direction.

Figure $15 \mathrm{a}$ reports other results obtained with the test trajectory \#3 with map errors. The intervals where PLs in the CT direction show an increase correspond to roundabouts. In this experiment, some map errors were automatically detected by the system. Figure $15 \mathrm{~b}$ shows the residuals corresponding to the left camera measurements. From sampling instants 931 to 2278 , the residual $r_{\text {cameraL2 }}$ indicates that the measurements $C_{0, \text { left,2 }}$ is erroneous, while $r_{\text {cameraL1 }}$ indicates that $C_{0, \text { left, } 1}$ is error-free. As discussed in section $\mathrm{V}$, the error comes from the map. This was verified afterwards by comparing manually the environment and the map: the camera detected two markings on the left side, while only one features in the HD map. Despite map errors, the filter provides $P L s$ that are always reliable. 
Table II

TUNing Stage APPlied to THE TRAINING TRAJECTORIES. MEASURED IR IN THE AT AND CT DIRECTIONS FOR A TIR=10 ${ }^{-3}$. IN YELLOW, THE NUMBERS CONSIDERED AS IMPORTANT FOR THE CHOICE OF DOF.

\begin{tabular}{|c|c|c|c|c|}
\hline dof & $\begin{array}{l}\text { Trajectory \#1 } \\
\left(\times 10^{-3}\right)\end{array}$ & $\begin{array}{c}\text { Trajectory } \\
\# 2 \\
\left(\times 10^{-3}\right)\end{array}$ & $\begin{array}{c}\text { Trajectory } \\
\# 3 \\
\left(\times 10^{-3}\right)\end{array}$ & $\begin{array}{l}\text { Mean value } \\
\left(\times 10^{-3}\right)\end{array}$ \\
\hline \multicolumn{5}{|c|}{ Measured IR in the AT direction } \\
\hline$v=4$ & 0 & 0 & 0 & 0 \\
\hline$v=5$ & 0 & 1 & 0 & 0.33 \\
\hline$v=6$ & 0 & 131 & 2.1 & 44 \\
\hline$v=8$ & 0 & 411 & 62 & 158 \\
\hline$v=9$ & 0 & 508 & 77 & 195 \\
\hline$v=10$ & 0 & 582 & 86 & 223 \\
\hline$v=100$ & 0.08 & 857 & 174 & 344 \\
\hline \multicolumn{5}{|c|}{ Measured IR in the CT direction } \\
\hline$v=4$ & 0 & 0 & 0 & 0 \\
\hline$v=5$ & 0 & 0 & 0 & 0 \\
\hline$v=6$ & 0 & 0 & 0.37 & 0.12 \\
\hline$v=8$ & 0 & 0 & 2.1 & 0.7 \\
\hline$v=9$ & 0 & 0 & 2.6 & 0.87 \\
\hline$v=10$ & 0.32 & 0.061 & 3.4 & 1.26 \\
\hline$v=100$ & 1.9 & 10.5 & 10 & 7.5 \\
\hline
\end{tabular}

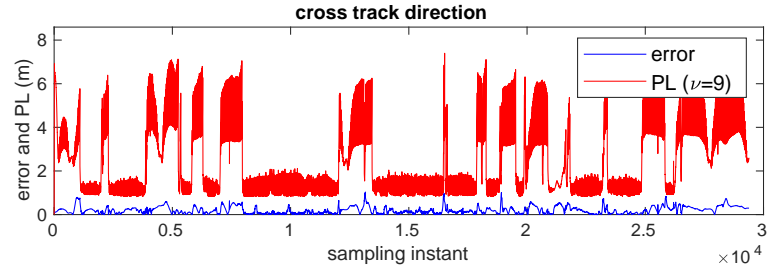

along track direction

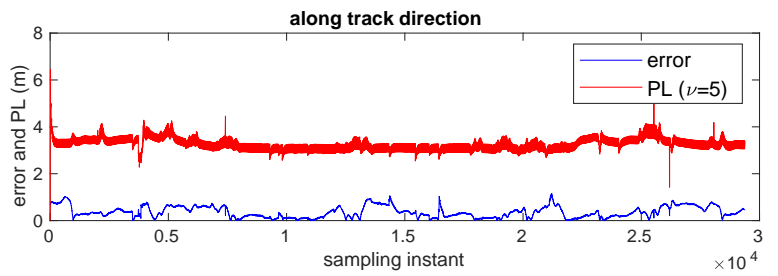

Figure 12. PLs after the FDE step in the AT and CT directions. Test trajectory $\# 1$.

Table III

Test STAGE. MEASURED IR APPLIED to THE THREE TEST TRAJECTORIES.

\begin{tabular}{ccc}
\hline & \multicolumn{2}{c}{ Measured IR } \\
\hline Test Trajectories & AT & CT \\
& $(v=5)$ & $(v=9)$ \\
\hline Test trajectories \#1,\#2 and \#3 & 0 & $0.5 \times 10^{-3}$ \\
\hline
\end{tabular}

Finally, it should be noted that using a Gaussian distribution for the data fusion is a good approach, since the Gaussian distribution models the central part well. Since the test statistics are created according to the filter state estimates (considered

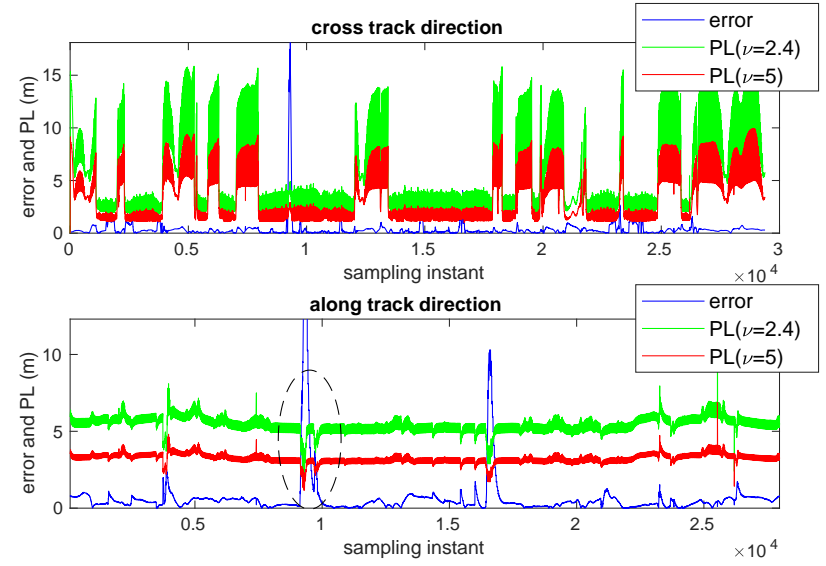

Figure 13. PLs without FDE in the AT and CT directions for two Student's $t$-distributions $(v=5$ and $v=2.4)$. Test trajectory \#1.

as Gaussian), we retained the Chi-squared distribution for threshold setting. For the FDE, the false alarm probability is set to 0.05 , which is large enough to be handled well by a Gaussian distribution. However, this would not be the case if the false alarm probability was set to $10^{-3}$.

\section{CONCLUSION AND FUTURE WORK}

In this work, a general localization method for utilizing multi-lane marking detection with integrity considerations was proposed. It was tested using a GNSS receiver, DR measurements, a camera and an HD map. An FDE strategy based on a bank of information filters was used to determine the origin of 

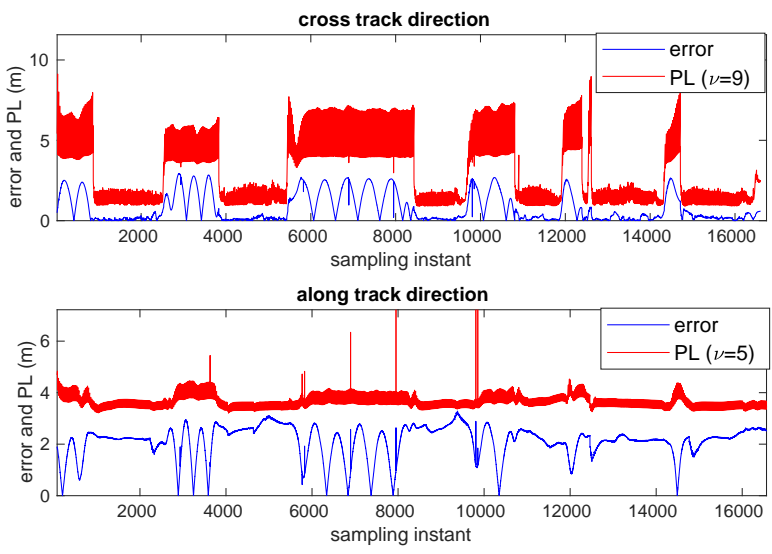

Figure 14. Test trajectory \#2 :PLs in the AT and CT directions.

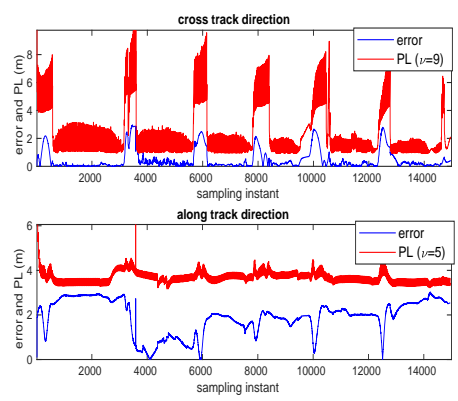

(a)

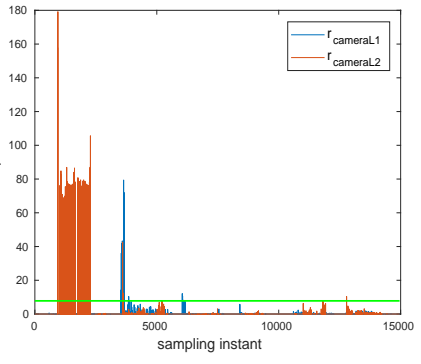

(b)
Figure 15. Test trajectory \#3: (a) PLs in the AT and CT directions, (b) left side camera residual with map errors visible at the beginning.

the faults thanks to a set of residual tests. We then proposed an accompanying method for bounding the errors, by replacing the Gaussian distribution with a Student's $t$-distribution. Our experimental results clearly show that this approach for $P L$ computation is more relevant than classical methods that use Gaussian distributions. The protection levels are computed in the AT and CT directions in order to give a concrete meaning of $P L$ for classical intelligent vehicle navigation tasks.

The performance of the approach was tested on different trajectories. The results obtained demonstrate the importance of the FDE stage and the characterization of the positioning errors by a heavy-tailed law for bounding errors when some faulty measurements remain in the computation process after the FDE step. Under the conditions of our experimental trials, we concluded that a Student's $t$-distribution with 9 dof is required in the CT direction, while 5 is required in the AT direction. We believe that the proposed method can be used in different land navigation environments. To adjust the dof of the $t$-distribution, data acquisitions with a ground truth system are necessary in the target environment (urban, suburban, open sky, etc.). This is in line with the strategy for deploying level 4 SAE autonomous vehicles within a well-defined operational design domain.

\section{ACKNOWLEDGMENT}

This work was carried out within SIVALab, joint laboratory between Renault and Heudiasyc UMR UTC/CNRS.

\section{REFERENCES}

[1] P. B. Ober, "Integrity prediction and monitoring of navigation systems.," 2004.

[2] N. Zhu, J. Marais, D. Betaille, and M. Berbineau, "GNSS position integrity in urban environments: A review of literature," IEEE Transactions on Intelligent Transportation Systems, vol. PP, no. 99, pp. 1-17, 2018, ISSN: 1524-9050.

[3] P. Zabalegui, G. De Miguel, A. Pérez, J. Mendizabal, J. Goya, and I. Adin, "A review of the evolution of the integrity methods applied in gnss," IEEE Access, vol. 8, pp. 45 813-45 824, 2020.

[4] D. Betaille and R. Toledo-Moreo, "Creating enhanced maps for lanelevel vehicle navigation," IEEE Transactions on Intelligent Transportation Systems, vol. 11, no. 4, pp. 786-798, 2010.

[5] M. A. Quddus, W. Y. Ochieng, and R. B. Noland, "Current mapmatching algorithms for transport applications: State-of-the art and future research directions," Transportation research part c: Emerging technologies, vol. 15, no. 5, pp. 312-328, 2007.

[6] A. Kasmi, D. Denis, R. Aufrere, and R. Chapuis, "Map matching and lanes number estimation with openstreetmap," in 2018 21st International Conference on Intelligent Transportation Systems (ITSC), IEEE, 2018, pp. 2659-2664.

[7] Z. Tao, P. Bonnifait, V. Fremont, and J. Ibanez-Guzman, "Mapping and localization using GPS, lane markings and proprioceptive sensors," in IEEE/RSJ International Conference on Intelligent Robots and Systems (IROS 2013), 2013, pp. 406-412.

[8] M. Tossaint, J. Samson, F. Toran, J. Ventura-Trasvet, M. HernandezPahares, J. M. Juan, J. Sanz, and P. Ramos-Bosch, "The stanford ESA integrity diagram: A new tool for the user domain SBAS integrity assessment," Navigation, vol. 54, no. 2, pp. 153-162, 2007.

[9] J. Cosmen-Schortmann, M. Azaola-Saenz, M. A. Martinez-Olague, and M. Toledo-Lopez, "Integrity in urban and road environments and its use in liability critical applications," in Position, Location and Navigation Symposium, 2008 IEEE/ION, IEEE, 2008, pp. 972-983.

[10] M. Worner, F. Schuster, F. Dolitzscher, C. G. Keller, M. Haueis, and K. Dietmayer, "Integrity for autonomous driving: A survey," in Position, Location and Navigation Symposium (PLANS), 2016 IEEE/ION, IEEE, 2016, pp. 666-671.

[11] P. B. Ober, R. Farnworth, E. Breeuwer, and D. van Willigen, "Overbounding the SBAS integrity equation," Integricom [online]. Integricom, PB Ober, 2011.

[12] K. A. B. Ahmad, M. Sahmoudi, and C. Macabiau, "Characterization of GNSS receiver position errors for user integrity monitoring in urban environments," in ENC-GNSS 2014, European Navigation Conference, 2014.

[13] RTCA Special Committe-159, "Minimum operational performance standards for global positioning system/wide area augmentation system airborne equipment," Document No. RTCA/DO-229B, vol. 6, 1999.

[14] I. Nikiforov and B. Roturier, "Advanced raim algorithms: First results," Proceedings of the ION GNSS 2005, 2005.

[15] B. W. Parkinson and P. Axelrad, "Autonomous GPS integrity monitoring using the pseudorange residual," Navigation, vol. 35 , no. 2 , pp. $255-274,1988$

[16] M. A. Sturza, "Navigation system integrity monitoring using redundant measurements," Navigation, vol. 35, no. 4, pp. 483-501, 1988.

[17] Y. C. Lee, "Analysis of range and position comparison methods as a means to provide GPS integrity in the user receiver," in Proceedings of the 42nd Annual Meeting of the Institute of Navigation, 1986, pp. 1-4.

[18] R. G. Brown and P. McBurney, "Self-contained GPS integrity check using maximum solution separation," Navigation, vol. 35 , no. 1, pp. 41-53, 1988.

[19] R. G. Brown, "A baseline GPS RAIM scheme and a note on the equivalence of three RAIM methods," Navigation, vol. 39, no. 3, pp. 301-316, 1992.

[20] M. Brenner, "Integrated GPS/inertial fault detection availability," Navigation, vol. 43, no. 2, pp. 111-130, 1996.

[21] O. Le Marchand, P. Bonnifait, J. Ibañez-Guzmán, D. Betaille, and F. Peyret, "Characterization of gps multipath for passenger vehicles across urban environments," ATTI dell'Istituto Italiano di Navigazione, no. 189, pp. 77-88, 2009. 
[22] J. Liu, M. Lu, X. Cui, and Z. Feng, "Theoretical analysis of RAIM in the occurrence of simultaneous two-satellite faults," IET Radar, Sonar \& Navigation, vol. 1, no. 2, pp. 92-97, 2007.

[23] B. Roturier, E. Chatre, and J. Ventura-Traveset, "The SBAS integrity concept standardised by ICAO-application to EGNOS," NAVIGATION-PARIS-, vol. 49, pp. 65-77, 2001.

[24] K. Ali, F. Dovis, and M. Pini, "Characterizing local effects on protection level concept in urban environments," 2012.

[25] K. Ali, M. Pini, and F. Dovis, "Measured performance of the application of EGNOS in the road traffic sector," GPS solutions, vol. 16, no. 2, pp. 135-145, 2012.

[26] J. Marais, M. Berbineau, and M. Heddebaut, "Land mobile GNSS availability and multipath evaluation tool," IEEE transactions on vehicular technology, vol. 54, no. 5, pp. 1697-1704, 2005.

[27] U.S. Department of transportation, Instrument Procedures Handbook. Federal Aviation Administration, 2017, ISBN: 9781546337799.

[28] A. Nowak and C. Specht, "Snapshot RAIM algorithms availability in urban areas," Annual of Navigation, pp. 73-88, 2006.

[29] D. Salos, A. Martineau, C. Macabiau, B. Bonhoure, and D. Kubrak, "Receiver autonomous integrity monitoring of GNSS signals for electronic toll collection," IEEE transactions on intelligent transportation systems, vol. 15, no. 1, pp. 94-103, 2014.

[30] D. Margaria and E. Falletti, "The local integrity approach for urban contexts: Definition and vehicular experimental assessment," Sensors, vol. 16 , no. 2, p. 154, 2016.

[31] _ "A novel local integrity concept for GNSS receivers in urban vehicular contexts," in Position, Location and Navigation SymposiumPLANS 2014, 2014 IEEE/ION, IEEE, 2014, pp. 413-425.

[32] — "Proof-of-concept of the local integrity approach: Prototype implementation and performance assessment in an urban context," in Localization and GNSS (ICL-GNSS), 2015 International Conference on, IEEE, 2015, pp. 1-6.

[33] P. F. N. Madrid, Device and method for computing an error bound of a kalman filter based gnss position solution, US Patent App. 14/885,642, 2016.

[34] N. M. Pedro F., L. S. Maria D., and R. M. Miguel M., "New approach for integrity bounds computation applied to advanced precise positioning applications," presented at the Proceedings of the 28th International Technical Meeting of The Satellite Division of the Institute of Navigation (ION GNSS+ 2015), 2015.

[35] M. A. Saenz, Method for autonomous determination of protection levels for gnss positioning based on navigation residuals and an isotropic confidence ratio, US Patent App. 12/429,687, 2009.

[36] M. Azaola, D. Calle, A. Mozo, and R. Piriz, "Autonomous isotropybased integrity using GPS and GLONASS," presented at the Proceedings of the 23rd International Technical Meeting of The Satellite Division of the Institute of Navigation (ION GNSS 2010), 2010, pp. $2135-2147$.

[37] M. A. Quddus, W. Y. Ochieng, and R. B. Noland, "Integrity of mapmatching algorithms," Transportation Research Part C: Emerging Technologies, vol. 14, no. 4, pp. 283-302, 2006.

[38] M. Pang and C. Tiberius, "Precision and reliability of tightly coupled ppp gnss and landmark monocular vision positioning," Sensors, vol. 20, no. 5, p. 1537, 2020.

[39] R. Toledo-Moreo, D. Betaille, and F. Peyret, "Lane-level integrity provision for navigation and map matching with GNSS, dead reckoning, and enhanced maps," IEEE Transactions on Intelligent Transportation Systems, vol. 11, no. 1, pp. 100-112, 2010.

[40] F. Li, P. Bonnifait, and J. Ibanez-Guzman, "Map-Aided DeadReckoning With Lane-Level Maps and Integrity Monitoring," IEEE Transactions on Intelligent Vehicles, vol. 3, no. 1, pp. 81-91, Mar. 2018.

[41] M. S. Braasch, "Multipath effects," Global Positioning System: Theory and Applications., vol. 1, pp. 547-568, 1996.

[42] P. D. Groves, Z. Jiang, L. Wang, and M. K. Ziebart, "Intelligent urban positioning using multi-constellation GNSS with 3d mapping and NLOS signal detection," 2012.

[43] J. Al Hage and M. E. El Najjar, "Improved outdoor localization based on weighted kullback-leibler divergence for measurements diagnosis," IEEE Intelligent Transportation System Magazine, vol. Early access, 2018.

[44] D. Betaille, S. Miquel, F. Godan, and F. Peyret, "3d-city-model-aided gnss accurate positioning with integrity provision using simplified geometry of buildings," in European Navigation Conference 2015, 2015.
[45] A. Dhital, J. B. Bancroft, and G. Lachapelle, "A new approach for improving reliability of personal navigation devices under harsh gnss signal conditions," Sensors, vol. 13, no. 11, pp. 15 221-15 241, 2013.

[46] S. Kotz and S. Nadarajah, Multivariate T-Distributions and Their Applications. Cambridge university, Feb. 2004.

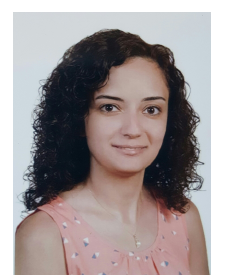

Joelle Al Hage has an engineer's degree in Electrical and Electronic Engineering (specialty Automatic Control and Industrial Computing) and a master's degree in Reliability, Identification and Diagnosis from the Lebanese University (2013). In 2016, she was awarded her Ph.D. degree in Automatic and Computer Science by the University of Lille in France. From 2017 to 2019 she was a CNRS researcher at SIVALab (a joint Renault-UTC-CNRS laboratory). Since 2019 she has been an associate professor in the Computer Science and Engineering Department at UTC and is carrying out research in the Heudiasyc laboratory (UMR 7253, UTC/CNRS). She remains a member of SIVALab. Her research is focused on multi-sensor data fusion with fault tolerance, the integrity of localization and perception methods for autonomous vehicles, and collaborative localization

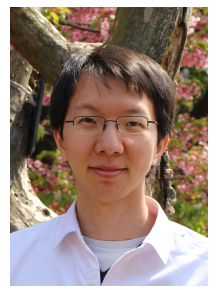

Philippe Xu received his B.Sc. and M.Sc. degrees in Computer Science from the Ecole Normale Supérieure de Cachan, France, in 2009 and 2011, respectively. He was awarded his $\mathrm{Ph} . \mathrm{D}$. degree by the Université de Technologie de Compiègne (UTC), France, in 2014. Since 2015 he has been an associate professor in the Computer Science department of UTC and is carrying out his research in Heudiasyc UMR 7253 (UTC/CNRS). Since 2017 he has also been a member of SIVALab, a joint Renault-UTCCNRS laboratory. His research interests are information fusion, computer vision, and machine learning applied to autonomous vehicles.

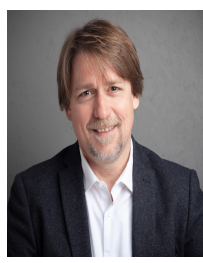

Philippe Bonnifait received his $\mathrm{Ph} . \mathrm{D}$. in Automatic Control and Computer Science from the Ecole Centrale de Nantes in France in 1997. He is currently a professor in the Computer Science and Engineering Department, Université de Technologie de Compiègne, France. Since 1998 he has been part of Heudiasyc UMR 7253, a joint UTC-CNRS research laboratory. His research interests include intelligent vehicles, and high-integrity positioning and map-matching for autonomous navigation in structured outdoor environments.

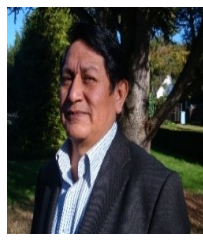

Javier Ibanez-Guzman received his Ph.D. from the University of Reading on a SERC fellowship, and an MSEE from the University of Pennsylvania (USA) as a Fulbright scholar. In 2011 he was visiting scholar at the University of California, Berkeley. $\mathrm{He}$ is member of the technical staff at Renault S.A., working on intelligent vehicle technologies. Formerly he was senior scientist at a SimTech (Singapore), where he spearheaded work on autonomous ground vehicles. He is an IEEE Member, C.Eng. (UK) and Fellow of the Institute of Engineering 